\title{
A layered modular polymeric $\mu$-valve suitable for lab-on-foil: design, fabrication, and characterization
}

\author{
Allwyn Boustheen • F. G. A. Homburg • \\ M. G. A. M. Somhorst • Andreas Dietzel
}

Received: 11 April 2011/Accepted: 31 May 2011/Published online: 7 July 2011

(C) The Author(s) 2011. This article is published with open access at Springerlink.com

\begin{abstract}
Cost-effective fabrication of microfluidic networks require that all components have to be manufactured with up-scalable processes such as reel-to-reel fabrication of foil-based devices. A microvalve design must take into account functional requirements together with manufacturing feasibilities. Here we present the development of a modular polymeric laser structured microvalve. The complete valve structure is designed to be used in a bendable lab-in-foil system. The modular microvalve design consists of three layers: an actuator layer, an interfacing membrane, and a passive microchannel layer to be separately fabricated and then stacked. Different actuator layer concepts are compared out of which a thermal actuation scheme generating sufficient stroke using phase changing paraffin is chosen. The passive layer is designed with a shallow and sufficiently smooth spherical cavity that acts as the valve seat from which paraffin material can reliably retract during solidification. The shape and dimensions of the shallow cavity are derived from the natural membrane deflection and from the channel cross section. It is not essential that all the paraffin within the actuator cavity to be molten for valve closure allowing a high degree of assembly tolerance and inherent sealing of actuator cavity. All the module layers in the current prototype are structured using 3D laser
\end{abstract}

Electronic supplementary material The online version of this article (doi:10.1007/s10404-011-0832-3) contains supplementary material, which is available to authorized users.

A. Boustheen $(\bowtie)$ · F. G. A. Homburg · M. G. A. M. Somhorst Eindhoven University of Technology, Den Dolech 2, 5600MB Eindhoven, The Netherlands

e-mail: a.boustheen@tue.nl

A. Dietzel

Holst Center, System-in-foil Program, High Tech Campus, Eindhoven, The Netherlands fabrication processes but mass-fabrication methods like reel-to-reel hot-embossing are foreseen as well. A prototype microvalve stack was assembled with a thickness of $1.1 \mathrm{~mm}$ which could be further reduced to meet the requirements of extremely flexible lab-on-foil systems. The closed valve is tested up to a pressure of $3 \mathrm{kPa}$ without any measurable leakage. The dynamics of valve closure is evaluated by a new optical characterization method based on image processing of color micrograph sequences taken from the transparent valve.

Keywords Layered microvalve - Laser ablation · Lab-in-foil · Optical image characterization

\section{Introduction}

Passive components (channels or other small fluid containers, sieves) and the active fluidic components (valves, pumps, mixers, active filters, sensors) have to be integrated to form a micro total analysis system (Abgrall and Gué 2007). Extensive work on microvalves and micropumps that can control and manipulate fluids is described in the literature (Oh and Ahn 2006; Laser and Santiago 2004; Woias 2005; Iverson and Garimella 2008). For the successful transition from laboratory applications to industrial products both the passive elements and the active elements should be cost effectively integrated and form a robust system. Not only the component design but also the manufacturing processes have to allow design freedom in order to easily accommodate design variations. Manufacturing techniques such as reel-to-reel processing, micro injection molding and hot-embossing can be scaled up to produce a large number of devices made of polymeric material. Research has been published on multilayer fluidic systems in which more than 
one layer has been stacked up to create fluidic structures (Vig et al. 2011; Patrick Abgrall et al. 2006; Han et al. 2007; Flachsbart et al. 2006). Up to now these devices are constructed of one material and they only have passive fluidic structures. However, systems allow more elaborate functions if active elements are incorporated. Electrically addressable elements have the advantage of simple control signal pathways. Electrically and pneumatically addressable microvalves made from expensive materials like glass and silicon with complex processing steps have been reported in the literature (Oh and Ahn 2006; Song et al. 2008; Kaigala et al. 2008; Yang and Lin 2007). These will not be very suitable to ramp up to cost effective mass-production. Also a polymer layer based microvalve system has been recently reported (Pitchaimani et al. 2009) employing a liquid (fluorinert) as thermal actuation medium. However, in order to decrease the actuator dimensions and to increase actuation speed, a phase changing actuation medium with higher thermal expansion would be advantageous. Phase changing materials such as wax, and PEG (polyethylene Glycol) have been discussed for microvalve actuation in the literature (Pal et al. 2004; Kaigala et al. 2008). Paraffin based systems have been explored more than any other phase change systems and have been used in applications such as actuators, braille cells, micropumps and microvalves (JS Lee and Lucyszyn 2005; Selvaganapathy et al. 2003; Klintberg et al. 2003; Boden et al. 2006; Yoo et al. 2007). These systems are built on structural materials like polymers, glass and silicon however a pure polymeric microvalve has not been discussed yet. Described in plane actuator dimensions are typically large, not less than $2 \mathrm{~mm}$. The chamber accommodating the landing of the membrane in all these presented works is either circular or rectangular shaped with steep side edges. For using such materials, it is essential that the valve design can accommodate the transition between liquid and solid phase without inhomogeneous solidification. A sharply shaped rectangular channel will be difficult to completely retract from when the valve opens. Hence it is essential to develop a special microvalve design for thermal phase change actuation that is also compliant with up-scalable manufacturing flexibility. Systems-in-foil are receiving a lot of attention because of their robustness, multi-functionality, and reel-to-reel manufacturability (Vig et al. 2011; Brand et al. 2008). The integration of microfluidic functionalities in such systems made on flexible foil substrates offers a new concept named lab-on-foil (Focke et al. 2010). In this article a novel polymeric microvalve employing thermal phase change actuation is described. It is assembled from flexible foil layers and essential parts of the microvalve are structured using laser prototyping processes. Moreover methodologies have to be found to investigate the transient non equilibrium states of such a thermally driven valve. Therefore, we also present a novel microscopic technique to characterize the dynamic performance of an optically transparent valve structure.

\section{Actuation concepts}

The microvalve shall form a basic component to be used at several places within a thin planar lab-on-foil system, imposing dimensional constraints on the microvalve. The out of plane dimensions of the actuator should therefore not exceed $0.5 \mathrm{~mm}$. The system is envisioned to be built onto a network of channels and reservoirs. A planar valve diameter of approximately $1 \mathrm{~mm}$ would still allow to incorporate a number of microvalves within a microfluidic network. The cross-section dimensions of such channel network will typically be around $50 \times 50 \mu \mathrm{m}^{2}$.

\section{Valve performance targets}

The performance requirements for the valve actuator can be formulated based on four important criteria: flow rate, stroke, opening and closing times, and electrical input. The flow rate that can be controlled by the valve is determined by the differential pressure between in- and out-flow and by the channel dimensions. With the mentioned channel dimensions (in laminar flow regime) a pressure difference between 3 and $300 \mathrm{kPa}$ seems a realistic assumption (corresponding to a flow of $1-100 \mu \mathrm{l} / \mathrm{s}$, respectively). A stroke in the range of $50 \mu \mathrm{m}$ is required to close the channel completely. Electrical input signals should not be in conflict with device usability and therefore voltages are assumed not to exceed $500 \mathrm{~V}$. Higher voltages may lead to ionization of the fluid in the channels. Power consumption should not exceed $500 \mathrm{~mW}$ to allow for portability of the device. The performance of an actuator in relation to the dimensions can be described by work density. A range of $10^{1}-10^{3}\left(\mathrm{~J} / \mathrm{m}^{3}\right)$ was calculated for the required work densities with the defined constraints and the equation $F \cdot d / V$, where $F$ is the force that is required to close the valve (differential pressure for the targeted flow rate divided by in-plane area of the microvalve), $d$ is the targeted stroke, and $V$ is the volume of the actuator considering the dimensional constraints. A closing time of few milliseconds (around $100 \mathrm{~Hz}$ in terms of frequency of operation) would be ideal but for some applications (for example disposable actuate-once systems) a few seconds will also be sufficient.

\section{Comparison of actuation principles}

Five actuation principles, thermal, electrostatic, piezoelectric, electro active polymer (EAP), and shape memory 
are measured against the above performance requirements in the following analysis. Electromagnetic actuators are not considered since fabrication is complex and integration within the required dimensions is difficult. To allow a quantitative comparison of the actuation principles the actuator dimensions are kept almost fixed. Four relevant performance parameters: work density, maximum frequency, maximum force, and achievable stroke were derived in an earlier analysis of the physical principles
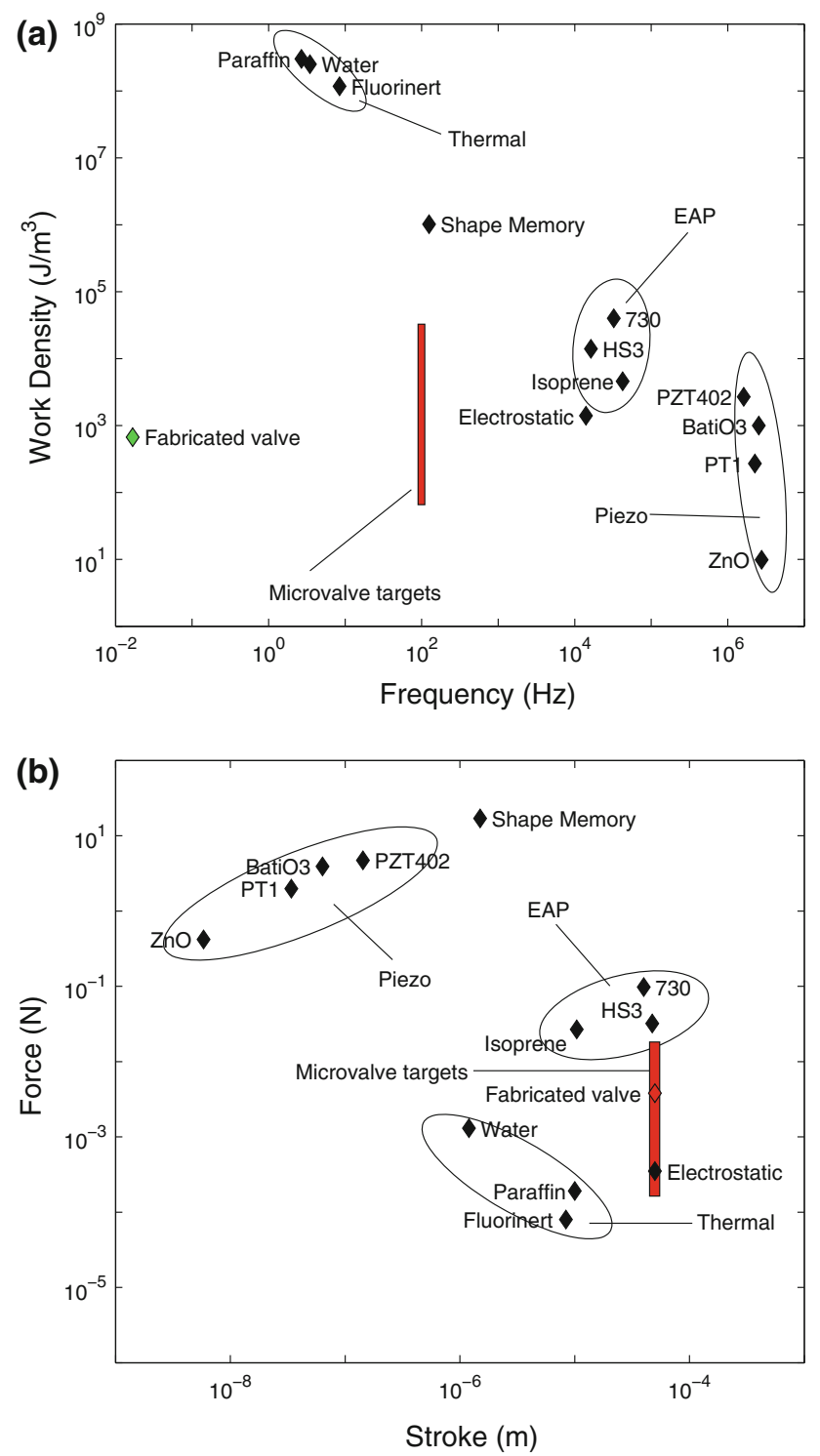

Fig. 1 Actuation performance in terms of a work density, and frequency, $\mathbf{b}$ force and stroke derived from basic physical principles and targeted microvalve dimensions. Each dot represents the numerical value for the calculated parameters and annotations represent the corresponding materials. Some dots are grouped in a blob for better comprehension. For comparison the microvalve performance targets are displayed as rectangles. Data taken from literature (Boustheen et al. 2008). The obtained force and work density values are also shown for the fabricated microvalve
(Boustheen et al. 2008). They are plotted in Fig. 1 along with the targeted microvalve performance parameters.

Piezoelectric actuators can deliver the desired performance in terms of work density and frequency. They deliver more than the desired force but not enough stroke. Electrostatic and electroactive polymer actuators can deliver or even surpass all the targeted values. Thermal actuators show more than sufficient work density and almost sufficient stroke but low force and low operating frequency. Shape memory actuators show high values in force, work density, and good match with the frequency requirements. They only fall short with respect to stroke.

With a mechanical transmission, a piezoelectric actuator can produce the necessary stroke. But this requires an additional assembly step, which is a disadvantage in view of industrial processes. Electrostatic actuators need high voltages and there is risk of ionizing the liquid to be controlled by the microvalve. Electroactive polymer actuators require high electric fields that usually translate in high voltages as well. However, a reduced applied voltage can be compensated by stacking several thin layers and thereby achieving the required stroke. Thermal actuators are slower than the other actuators but do not need an additional mechanical transmitter and can easily be separated from the fluid by a thin conformal membrane. Moreover thermal actuation can be made faster by further increasing the surface to volume ratio of the expanding material. Shape memory actuators though promising have fabricational and integration challenges. Moreover an additional training is required before successful operation. Electroactive actuators are also promising but require specific designs. For the investigations described in the following a thermal microvalve was chosen even though not all the requirements are fully met yet. However, the modular concept would potentially allow to exchange the active layer to use alternative actuation concepts as well.

\section{Microvalve design}

\subsection{Physical principle}

Paraffin undergoes significant non linear volumetric expansion during phase transition from solid to liquid. By confining the paraffin within a boundary consisting of stiff and compliant structures the thermal expansion can produce directed actuation. The resulting temperature distribution (without considering convection and radiation) for a heat flow in the system is given by

$\nabla^{2} T+\frac{\dot{q}}{k}=\frac{1}{\alpha} \frac{\partial T}{\partial t}$

where $k$ is the thermal conductivity, $\dot{q}$ is the rate of heat generation, $\alpha=k / \rho c_{p}(T)$ is the thermal diffusivity, and 

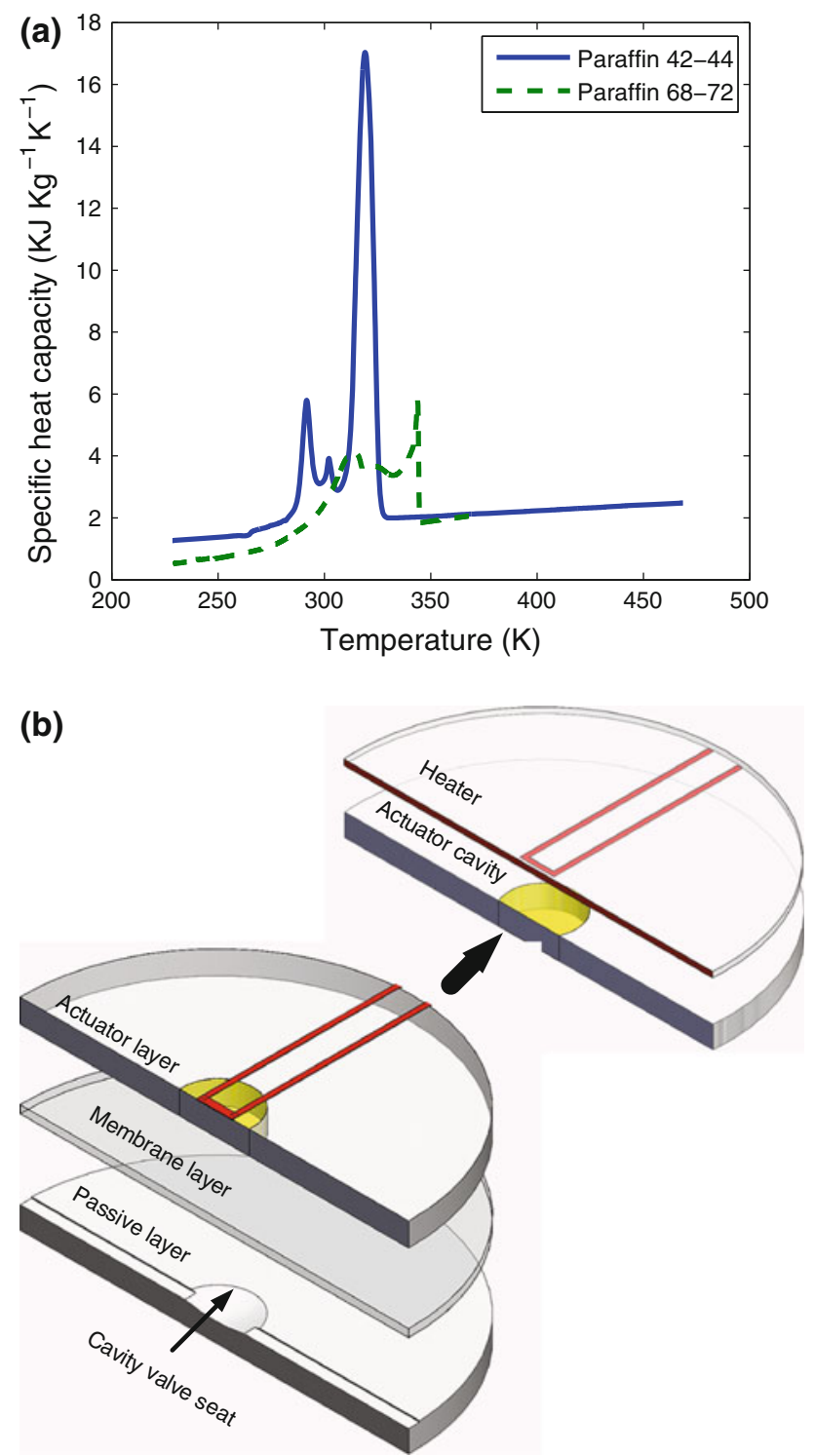

Fig. 2 a DSC scans of Paraffin $42-44$ and Paraffin 68-72. At temperatures below the melting temperatures solid to solid transitions can be observed as additional peaks. (b) Design schematic. The microvalve is divided into three module layers: active, passive, and membrane. The actuator layer itself consists of two foil layers: heater layer on a foil and a second foil containing the actuator material. In the later described prototype the actuator material is paraffin contained in a cavity

$c_{p}(T)$ is the specific heat at constant pressure. The material properties $k$ and $c_{\mathrm{p}}(T)$ influence the operational frequency and the amount of power generation required for the desired material temperature increase. During the phase transition the specific heat $c_{p}(T)$ of paraffin changes with respect to temperature resulting in change in volume and density. The heat of fusion is the integration of the specific heat capacity curve and is taken into account in determining the input power. The surface area of the system determines the heat losses in the system and therefore affects the operational frequency of the system. These parameters were analyzed in the previous work (Boustheen et al. 2008) and results of that were discussed in the previous discussion.

\section{Material selection}

Paraffins consist of one or more hydrocarbon $\left(\mathrm{C}_{n} \mathrm{H}_{2 n+2}\right)$ species and exist in crystalline form below their melting temperature. Different kinds of paraffin are characterized by varying hydrocarbon chain lengths. Variation in chain length leads to variation in melting temperatures ranging from 143.3 up to $388 \mathrm{~K}$. This results in a wide variety of materials having similar physical properties that can be tuned to operate at a desired temperature range. Moreover paraffin is in most cases inert and immiscible with water and has low thermal and electrical conductivity. The solid to liquid phase transition is accompanied by a volume change between 10 and 30\% (Selvaganapathy et al. 2003) that remains virtually unchanged even under pressures of $80 \mathrm{MPa}$ (Walsh and Zoller 1995). This makes paraffin attractive as a thermal actuation material. Two types of commercially available paraffin (from Merck KgaA, Germany) labelled as Paraffin 42-44 and Paraffin 68-72 were evaluated, with numbers denoting the solidification phase transition temperature interval. To evaluate their thermodynamic properties, DSC (Differential scanning calorimetry) scans were performed and are presented as specific heat capacity as function of temperature in Fig. 2a. Paraffins undergo solid to solid phase transitions in addition to solid to liquid transition. These are observed in the presented specific heat capacity curves. Paraffin 42-44 shows a sharp phase transition while the transition interval for paraffin $68-72$ is wider. The heat of fusion of the paraffin $42-44$ is calculated as $225 \mathrm{~kJ} \mathrm{~K}^{-1}$. Paraffin $42-44$ is used in the prototype because its sharper phase transition will make the valve close on a narrower temperature range.

Polycarbonate is a thermoplastic material that can be microstructured by excimer laser but also injection molded or hot embossed and hence was used as base material for the prototype valve. Platinum, gold, and copper are suitable as resistive materials that can form a microresistor to heat the paraffin. They all can be deposited by sputtering or evaporation. Gold has been chosen, since it was available in our lab. It has about the same temperature coefficient of resistance as copper and is not expensive as platinum. A membrane covering the expanding material is another important part of the system. The physical, mechanical, and chemical properties of this membrane determine the operation temperature and pressure range and the range of fluids that can be controlled. PDMS was chosen because it is considered in the literature as bio compatible (JN Lee 
et al. 2004) and it is an elastomer (low Young modulus) that can be easily deformed under pressure and will return to its initial shape as soon as the pressure is withdrawn. It is also stable in the expected operation range ( $\sim 293-353 \mathrm{~K})$.

\section{Microvalve design}

To allow independent manufacturing of each component a modular layer-based system is proposed. Microfabrication processes for plastics like laser ablation, micro injection molding and hot embossing can be relatively complex. A successful industrial production may require combination of different technologies based on the dimensional and functional features of the components (Pham et al. 2002). Therefore, a modular system in which all the structures are structured from only one side has a high potential for commercialization.

The entire prototype is made on flexible polycarbonate foils (except the membrane which is made up of PDMS) as depicted in Fig. 2b. The actuator layer consists of two sheets: a $500 \mu \mathrm{m}$ thick polycarbonate sheet acts as the base on which the heater is structured and the actuator cavity is formed in a $250-\mu \mathrm{m}$ thick polycarbonate sheet in which the paraffin is filled. The two polycarbonate sheets together form the actuator layer. Next, the membrane is superimposed on the actuator layer. The passive module layer consists of channels and fluidic interconnects to the system. The passive layer also has a valve seat structure to be precisely aligned with the actuation cavity so that the bending of the membrane is directed onto the valve cavity resulting in perfect valve closure.

In accordance with previously mentioned target dimensions of the microvalve an actuating cavity of $1100 \mu \mathrm{m}$ diameter is chosen. This diameter is larger than the spherical cavity diameter to enable closure of the valve in case of misalignment. A heater of nominal radius $450 \mu \mathrm{m}$ is designed to fit within the boundaries of the actuating cavity. From these dimensions, an estimation of the membrane deflection can be made. The shape of the paraffin volume added by expansion can be assumed as a spherical cap accommodated by the deflecting the PDMS membrane. This volume $V_{\text {cap }}$ is given by

$V_{\text {cap }}=\frac{1}{6} \pi h\left(3 a^{2}+h^{2}\right)$

where $a$ is the radius and $h$ is the height of the spherical cap, $a$ can be taken as $0.45 \mathrm{~mm}$. Assuming the expansion of the paraffin to be $10 \%$ (minimum paraffin expansion during complete solid to liquid phase change)(Selvaganapathy et al. 2003), $V_{\text {cap }}$ is obtained. Solving Eq. 3 and ignoring the stiffness of the membrane the deflection $h$ is estimated to be approximately $50 \mu \mathrm{m}$. This volume ( $\left.V_{\text {cap }}\right)$ is less than the total volumetric expansion of the paraffin within the actuator cavity thereby providing inherent sealing of actuator preventing paraffin from expanding onto the interfaces. The shape and surface of valve seat have to be such that the membrane will be able to smoothly land and perform complete valve closure. Moreover the valve seat geometry has to allow a complete retraction of a potentially incoherently solidifying material. Therefore, the shallow cavity is designed to have almost the calculated shape of the spherical cap (Eq. 3) with a deepest point of $50 \mu \mathrm{m}$ (allowing efficient channel dimensions of $30 \mu \mathrm{m}$ depth) and a diameter of $1 \mathrm{~mm}$. The shallow cavity has a flat bottom at the center, which reduces the volume that is ineffective for valve closure.

The microheater is designed to have serpentine shape within a circular boundary. Additional resistive leads were designed to sample the temperature of the heater in real time. This may be used for feedback control of the power supply to the heater. A PDMS membrane with thickness of $100 \mu \mathrm{m}$ or below will not influence valve closure since the pressure generated from the paraffin is much larger than the pressure required for membrane deflection.

\section{Fabrication}

The fabrication is carried out in separate module layers as previously mentioned and is explained in detail in the following.

\section{Laser micro-fabrication of the passive layer}

The developed laser processes are described here in detail not only because they were used to fabricate microvalve prototypes but also because they may be applied for fabricating master structures from which many replicas can be made in an industrially scaled process (Jensen et al. 2004). Reel to reel fabrication and replication processes for microfluidics are also described in literature (Vig et al. 2011). The passive layer is made in a polycarbonate sheet of thickness $250 \mu \mathrm{m}$ and lateral dimensions of $24 \times 20 \mathrm{~mm}^{2}$. Two through holes of $2 \mathrm{~mm}$ diameter act as external fluidic ports. A channel connects the two fluidic ports with the passive cavity in middle. The channel has steep straight walls and a curved bottom. The width of the channel is $250 \mu \mathrm{m}$ and the average depth is $30 \mu \mathrm{m}$. The structuring of the shallow cavity was done by Excimer laser (Micromaster, Optec s.a, laser source ATL SP 300i). The laser produces lightpulses of 4-6 ns with a wavelength of $248 \mathrm{~nm}$ and can operate at a maximum frequency of $300 \mathrm{~Hz}$. A precision $\mathrm{x} / \mathrm{y}$ stage can facilitate scanning contours. The cavity is fabricated by means of a number of concentric contours similar as 
described for microlens fabrication (Naessens et al. 2003). With the following assumptions the cavity profile can be calculated: (i) The laser fluence is constant over the beam diameter $\phi$ (ii) the pulse repetition frequency is constant during a single contour scan, and (iii) the contour writing velocity is constant implying constant number of pulses per written distance. For a contour diameter $D$, and a beam diameter $\phi$ the radial depth profile $y(r)$ of the cavity can then be approximated as (see Fig. 3a).

$y(r)=a \cdot p(r)$

where $p(r)$ is a shape function that gives relative ablation depths from a contour scan in dependence of the radial coordinate $r$ with origin at the center of the contour circle. The shape function is normalized to one. The maximum ablation depth in a contour of diameter $D$ is given by
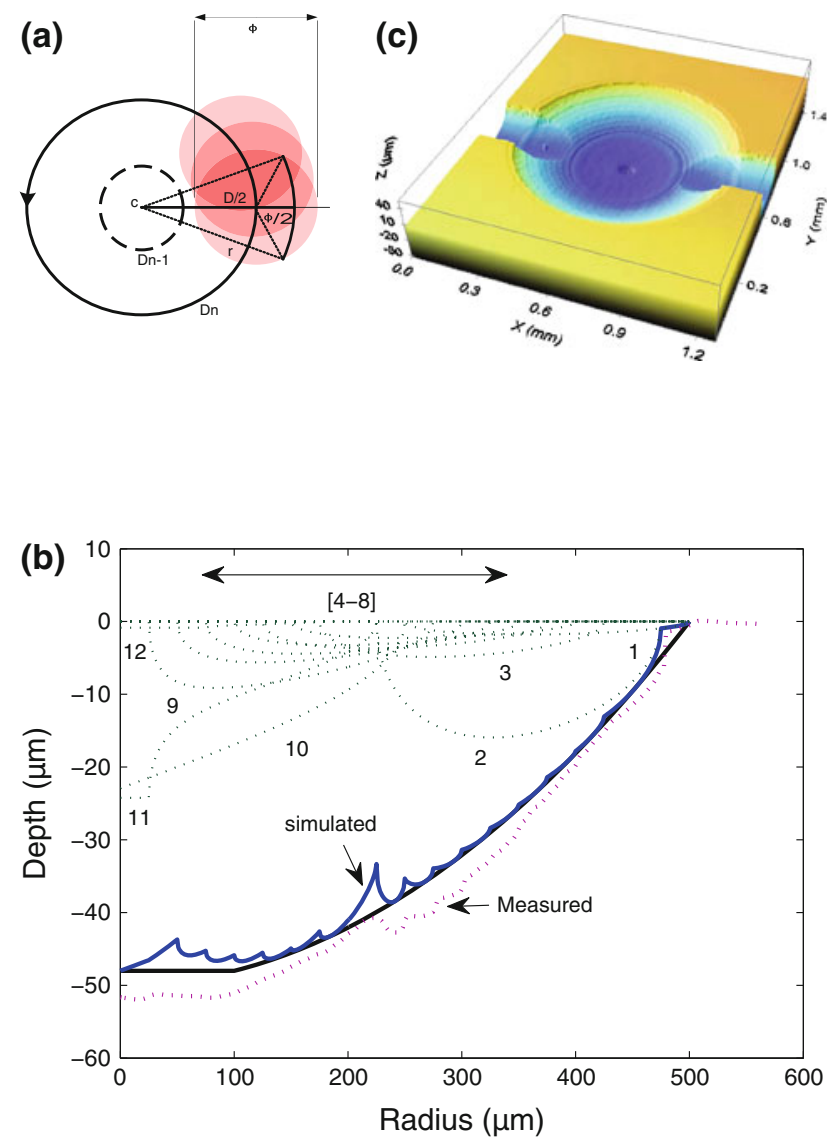

Fig. 3 a Schematic representation of the contour scanning method. b Laser ablation profiles for fabrication of the shallow and smooth valve cavity. The calculated individual ablation contours are annotated underneath each contour. Contours 1,2,3,9-12 are annotated individually. Contours 4-8 are annotated over the area where they appear. The desired spherical cavity profile with a flat bottom is displayed together with the optimized superposition of the 12 individual simulated ablation contours and a profile (dotted line) of a real cavity measured with an optical profilometer after real laser ablation. c The full 3D profile of the fabricated passive cavity obtained using a confocal profilometer (Sensofar Plu 2300) $a=\pi D k / v$ where $k$ is the ablation depth per unit time and $k$ can be written as $k=\Delta d \cdot f$ where $\Delta d$ is the ablation depth per pulse and $f$ is the pulse repetition frequency. The scanning velocity $v$ can be written as $v=(f / n) \cdot \phi$ where $n$ is the number of pulses that overlap at any location. Now $a$ can be written as

$a=\frac{\pi D n \Delta d}{\phi}$

and thereby relating the number of pulses and the velocity. The shape function can be described as

$p(r)=\left\{\begin{array}{cc}\frac{1}{\pi} \arccos \left(\frac{D^{2}+4 r^{2}-\phi^{2}}{4 r D}\right) & \text { if } \frac{D-\phi}{2}<r<\frac{D+\phi}{2} \\ 0 & \text { if } r<\frac{D-\phi}{2}, r>\frac{D+\phi}{2}\end{array}\right.$

Using Eq. 4 individual circular trench profiles can be calculated. By summing a number of circular trench profiles the desired cavity profile $y(r)$ can be obtained (Naessens et al. 2003). $y_{i} r_{i}$ are the individual trenches which are a result of $D_{i}$ representing the $i$ th contour diameter as sketched in Fig. 3a. The contour diameter and the distance between the successive contours together with beam diameter $\phi$ and the number of pulses for each contour determine the cavity shape and depth. A systematic solution is employed to find a combination of these parameters leading to the desired cavity profile which is a spherical cavity with flat base at the bottom as shown in Fig. 3 b.

The desired cavity, the beam diameter $\phi$ and the distance between the successive contours $\Delta D$ are fixed. Starting with a value that equals the final diameter of the passive cavity the contour diameter is decreased for each subsequent contour. The minimum contour diameters are limited by accuracy of the stage and as the contour overlaps itself along the radial direction. In this case the shape function $p(r)$ has a value of 1 . A search routine is employed to find the optimum velocity for each contour by minimizing the deviation in depth from the desired profile. Smaller values for $\phi$ and $\Delta D$ result in smoother profiles but for the laser used and for acceptable processing times the number of contours is kept around 12. The real fabricated cavity is shown in Fig. 3c. The $R_{\mathrm{a}}$ value for the surface roughness is determined from measured topographies by fitting cubic polynomial curve to a section of the cavity to be $0.19 \mu \mathrm{m}$. This roughness was not limiting the valve closure when the experiments were performed.

\section{Actuator fabrication}

The actuator fabrication is done in two steps: fabrication of heater sheet and of the actuator module sheet. The actuator 
cavity of $1.1 \mathrm{~mm}$ is cut by means of a powerful (Coherent Inc., Laser characteristics: $4.5 \mathrm{~W}$ power at $25,10-100 \mathrm{kHz}$ frequency, $15 \mathrm{~ns}$ pulse width at $10 \mathrm{kHz}$ ) frequency tripled $\mathrm{Nd}$ :YAG laser $(355 \mathrm{~nm})$. In contrast to the excimer laser $(248 \mathrm{~nm})$ for which polycarbonate has good absorption and the ablation is of photo chemical nature the Nd:YAG laser structuring process is a combination of photo chemical and thermal processes. This leads to debris and thermal stress and creates a bulge at the hole rim with a height of around $5 \mu \mathrm{m}$. However, no sealing related issues in the microvalve operation that will be later discussed were observed. Screw holes were also cut into both sheets for assembly purposes. $300 \mathrm{~nm}$ of gold is sputtered on the heater substrate and then patterned using the excimer laser to form the heater geometry.

Membranes were formed by spin coating uncured Sylgard 184 (PDMS) from Dow corning ${ }^{\text {TM }}$ over a polycarbonate substrate. The speed of the spin coating is adjusted to $1200 \mathrm{rpm}$ which yields (after a minute) a thickness of about $60 \mu \mathrm{m}$. After the curing of the PDMS the membrane can be peeled off.

\section{Microvalve assembly}

The finished module sheets are assembled in four steps to form a microvalve: (i) bonding of the heater and the actuator cavity (ii) filling of the actuator cavity with paraffin (iii) application of the membrane over the surface of the actuator cavity, and (iv) aligned placement of the passive layer over the already assembled stack. Since normal adhesives cannot be used for the bonding of PDMS a combination of bonding and clamping is used to build first prototypes. The adhesive problem can be addressed by modified bonding techniques (KS Lee and Ram 2009; Tang and Lee 2010; Vlachopoulou et al. 2009; Sunkara et al. 2011; Tennico et al. 2010) or PDMS interface bonding (Suk et al. 2008; Chow et al. 2006). The actuator is insensitive to bonding quality because not all the paraffin needs to be molten for closure of the valve and the remaining solid paraffin will seal the cavity. Mechanical clamping of the prototype allows us to apply sufficient pressure for the fluid flow in the channels not limited by delamination of bonded layers. An assembly stage with four degrees of freedom, $x, y, z$ and rotation angle in combination with a sample holder is used to allow precise alignment. One layer is placed on the stage and the other layer is placed on the sample holder with a finite separation between the two. After the layers are aligned under a microscope the $z$-axis is lowered and the upper layer is released from the vacuum tweezer.

The heater and the actuator cavity are bonded with solid adhesive sheets ( $3 \mathrm{M}$ 8212, acrylic based) of $50 \mu \mathrm{m}$ thicknesses which were patterned using excimer laser before protective layers were removed. Paraffin is melted on the actuator cavity by placing the system on a hot plate. After the paraffin has solidified, excess paraffin on top of the layer is scratched off with a sharp blade. The PDMS membrane is applied manually over the actuator layer. Finally, the passive layer is placed over the membrane and the assembly is completed by sandwiching the microvalve between plexiglas blocks clamped with screws.

\section{Characterization of microvalve operation}

Microvalving performance is verified and analyzed using two methods: (i) pressure-flow analysis and (ii) optical image intensity analysis. Pressure-flow characterization reveals the closure performance of the valve in thermal equilibrium for a certain pressure range. The analysis of color intensities in optical micrograph sequences is used to observe the transient states in the actuation process of the microvalve. Image analysis allows to characterize the dynamic performance of the valve actuation.

\section{Pressure-flow characterization}

Pressure difference is imparted using a hydrostatic head supplying DI water. Rhodamine B is used to dye the DI water to enable flow observation. The valve is connected to the hydrostatic pressure head and the external environment using capillaries of various diameters. When the valve is open, the output flow from the $250 \mu \mathrm{m}$ inner diameter capillary is weighed using a microbalance. When the valve is partially closed, the flow rate is low and is measured by observing the motion of the water meniscus in the capillary. The pressure-flow characteristics of the valve at three different power inputs (different currents through the heater) is shown in Fig. 4a. As the differential pressure is increased, the flow increases linearly as predicted by the Hagen-Poiseuille equation when the valve is open or partially open. Partial closure is indicated for the power of $20 \mathrm{~mW}$ as reduced flow compared with open state at $0 \mathrm{~mW}$. For the power of $25 \mathrm{~mW}$ the valve remains closed up to the maximum applied input pressure of $3 \mathrm{kPa}$. Further increase of pressures results in leakage at the layer interface and not through the valve seat when the current assembly setup is used.

\section{Dynamic performance characterization}

The microvalve is placed under a stereo microscope equipped with $1156 \times 1196$ pixel resolution camera to 
(a)

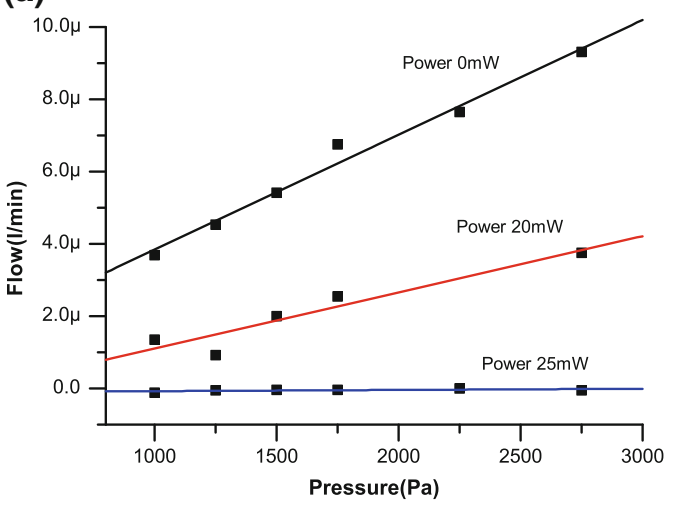

(b)

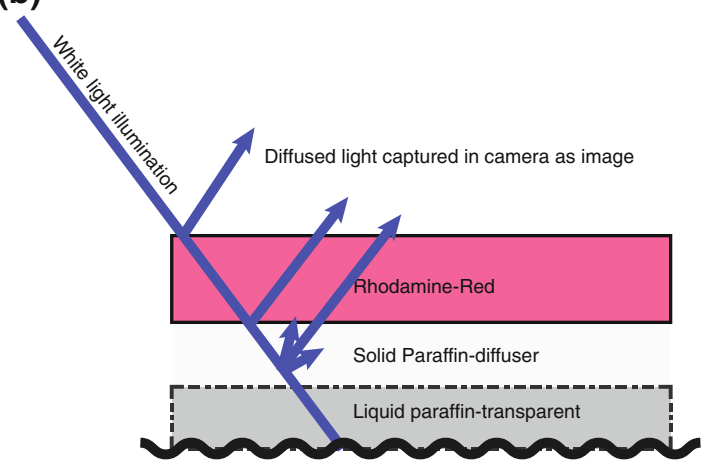

(c)

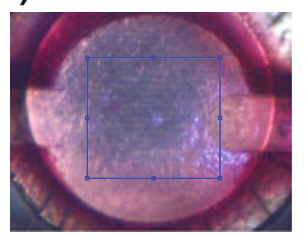

(d)

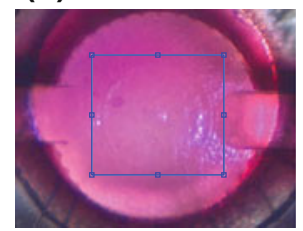

(e)

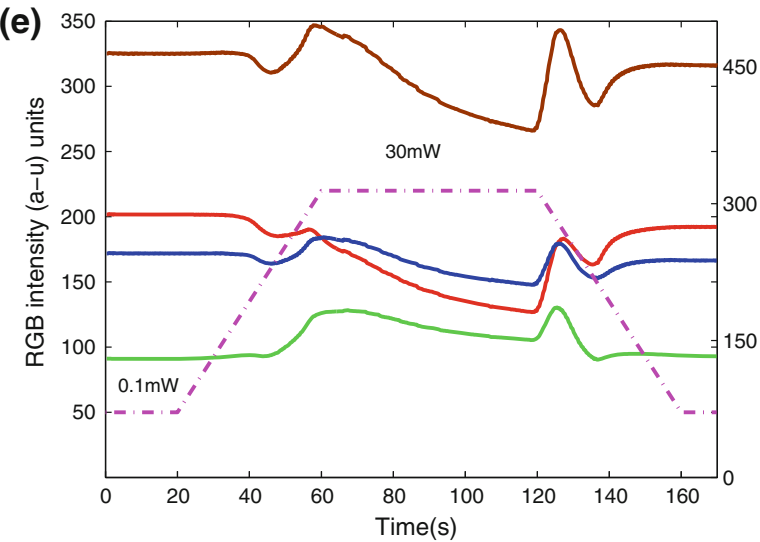

Fig. 4 Valve closure analysis: a The pressure valve characteristics for three different input power values. Valve is fully open when no input power is supplied, partial, and complete closure obtained for a power of 20 and $25 \mathrm{~mW}$, respectively. b Sketch of light back-scattering by the optical layers of the microvalve when operated with Rhodamine dyed water. The reflected light is captured by the microscope. $\mathbf{c}, \mathbf{d}$ shows the microvalve (diameter $1 \mathrm{~mm}$ ) in the closed and the open state, respectively. The bright area in the bottom of both pictures is due to the reflection from the slightly misaligned heater (gold-layer). The rectangles represent the area of the image analysis. e Red, green, and blue intensities and the cumulative

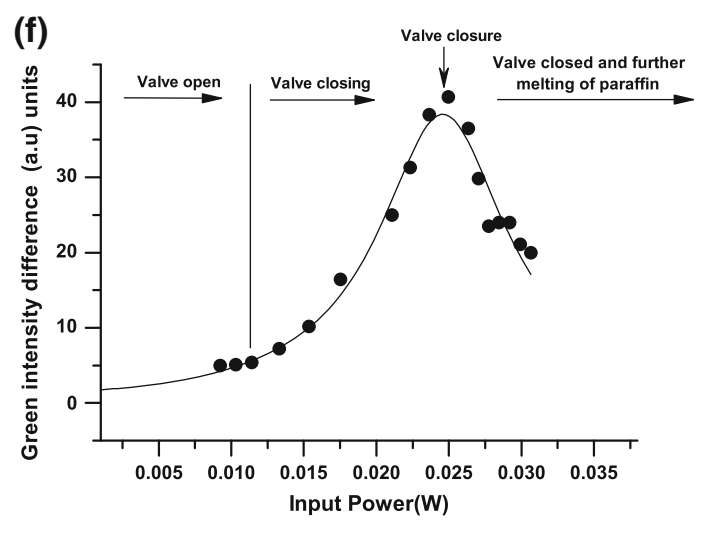

(g)

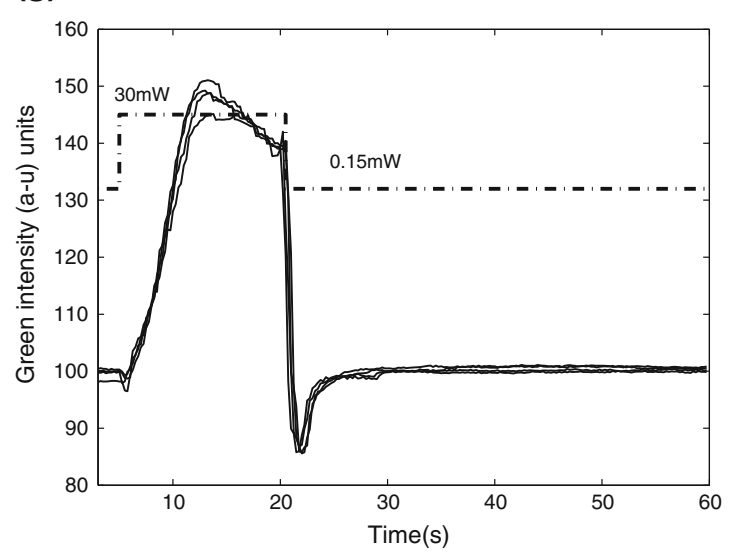

intensity values as function of time. The dotted line gives the heater current with annotations indicating the input power during actuation and nonactuation periods. $\mathbf{f}$ The increase of green intensities relative to the non powered state in dependence of operation power recorded after thermal equilibrium is reached. $\mathbf{g}$ The green intensity when the valve is operated at pulsed input signal of $30 \mathrm{~mW}$ as in (e).The more sudden changes in intensity after the current is switched are due to immediate changes in the transparency of the paraffin. Successive closing cycles are plotted in gray lines proving the repeatability of the system. The closing and the opening times of the valve is also obtained as 7 and $9 \mathrm{~s}$, respectively from the figure 
record color videos of the valve opening and closure. For the analysis the optical characteristics of the valve must be well-understood. Paraffin and the PDMS membrane are white or transparent. Rhodamine B (red/purple color) was used to dye the water. The layers are schematically represented in Fig. 4b. The Rhodamine filters and reflects red light. The Paraffin layer is white in color and acts as a diffuser, scattering back light that has passed the Rhodamine/water layer.

When the paraffin melts during the closure process it changes from being white to transparent scattering less light back into the microscope. Light from the illumination scattered back from the different layers contributes to the red, green, and blue in the microscopic images. The valve is activated by a power pulse as represented in Fig. 4e. The observed color changes are shown in Fig. 4c-e. Red color dominates when the valve is completely open. The red color component decreases when the valve closes because colored water leaves the cavity. In turn the blue and green intensities increase because the light filtering red colored water layer is reduced. From Fig. $4 \mathrm{e}$ it can be observed that the green intensity measured in the frames indicated in Fig. 4c, d follows the valve closure more directly than red and blue intensity components and hence seems most suitable for dynamical valve performance analysis. As the valve is powered the paraffin starts melting and partially becoming transparent. Owing to this additional optical effect the relative green intensity (and also blue and red intensities) reduces again slowly due to the fact that more light can pass to deeper layers and are reflected out of the system. As the current through to the valve heater is ramped back to zero, the paraffin starts to solidify from the top immediately giving rise to the light backscattered into the microscope and the intensities of all the three color components peaks. Further opening of the valve allows more colored water to flow back into the valve cavity which brings the intensities of colors back to the initial values where the red color dominates. Therefore, from the green intensity modulation information about cavity closure/opening as well as about paraffin phase changes can be retrieved. To identify the power at which the valve closes, changes of green intensity in dependence of different power levels were measured at thermal equilibrium as shown in Fig. 4f. As the valve starts to close, the green intensity increases. The intensity increase is non-linear and this behavior is expected due to the non-linear volume expansion of paraffin (causing cavity volume decrease) during melting. The green intensity reaches maximum at $25 \mathrm{~mW}$ and is interpreted as the power at which the valve completely closes. Further increase of power does not push more colored water out of the cavity but results in decrease of green intensity due to further paraffin melting as explained previously.
The valve can also be operated to have shorter opening and closing times if the input power is raised above the one required for closure in a pulsed input. The closure of the valve for a pulse of $15 \mathrm{~s}$ and for a power of $30 \mathrm{~mW}$ $(14 \mathrm{~mA})$ is shown in Fig. $4 \mathrm{~g}$. The opening and the closing times of the valve is obtained as 7 and $9 \mathrm{~s}$, respectively. The actuator volume is $0.23 \mu \mathrm{l}$. A closing time of $7 \mathrm{~s}$ for a similar device actuated by using a liquid medium (FC-40) with an actuator volume of $0.79 \mu \mathrm{l}$ has been reported in the literature (Pitchaimani et al. 2009) but only at power levels around $80 \mathrm{~mW}$. Moreover pressure difference at which the valve was characterized was lower $(9.5 \mathrm{~mm}$ hydrostatic head $\sim 100 \mathrm{~Pa}$ ) compared with flow rate up to $9 \mu \mathrm{l} / \mathrm{min}$ and a pressure of $3 \mathrm{kPa}$ achieved with our system. To emphasize the good repeatability of our device closing characteristics of four consecutive valve cycles are plotted. Powering the valve constantly for up to 20 min kept the valve closed confirmed by a static water meniscus in the outflow tube. During these measurements, the temperature across the microheater can also be measured. The input power could potentially be feedback controlled using the micoheater resistor.

\section{Conclusion}

A flexible modular layer based and laser structured microvalve was designed and fabricated. The individual layers of the microvalve are shown in Fig. 5. The layer-based design enables the components to be manufactured separately thereby enabling more independent design, processing, and material choices for each layer. From the systematic analysis that was presented in the first sections, the design of the valve can be tuned for required flow rate and pressure and also the switching times. In the valving characterization the valve closed under a pressure of $3 \mathrm{kPa}$ with an input power of $25 \mathrm{~mA}$. The opening and the closing time of the microvalve is obtained as 7 and $9 \mathrm{~s}$, respectively. Moreover an efficient microscopic characterization method is presented to analyze the closing an opening dynamics of the partially transparent microvalve. The color intensity variations over time indicate the in- and out-flow of dyed water into and from the valve cavity and the transient states of the melting/solidifying processes of the paraffin actuation medium. The obtained switching speed does yet meet the targeted performance but can be improved if thermal dissipation is more efficient as for instance can be realized by higher surface to volume ratio of the actuator medium. The main advantages of this layered concept are: (i) All the components are structured from only one side. This will be highly advantageous when transferred to industrial scale processes. This will help to produce microsystems at low cost. (ii) Different phase 


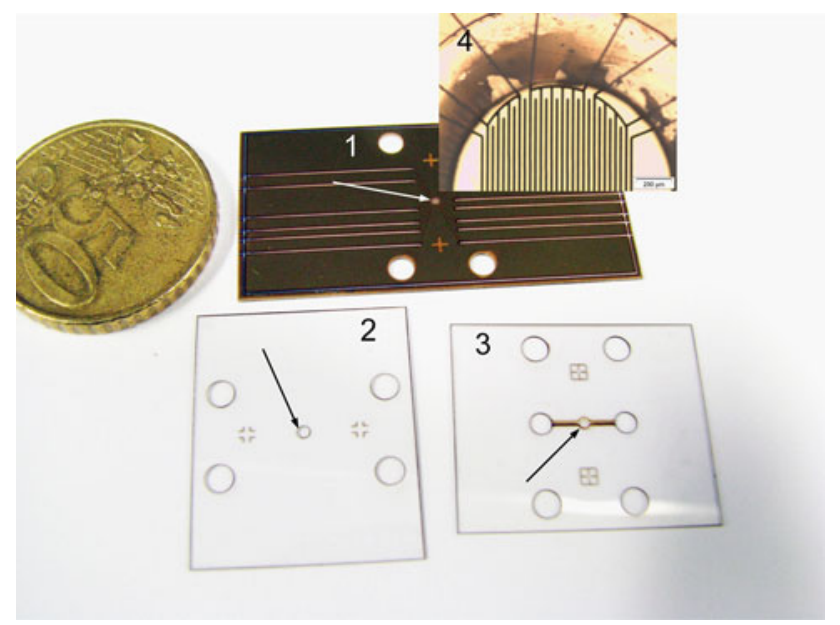

Fig. 5 The fabricated microvalve components except membrane. 1 Resistor layer, the actuating heater is in the center of this layer (see arrow) made by sputtered thin film of Au structured by excimer laser on a $500 \mu \mathrm{m}$ thick substrate. 2 Actuator cavity layer with thickness of $250 \mu \mathrm{m}$, cavity which is not yet filled with paraffin is indicated by an arrow. 3 Passive cavity sheet with channels and circular fluidic interface for the external connection. The circular valve seat is indicated by an arrow. 4 Microheater with assembled actuator cavity. Microheater meander can be seen in microscopic insert. For comparison, a coin of diameter $24 \mathrm{~mm}$ is also visible in the picture

changing paraffin materials can be selected based on the required volume of expansion and the required temperature regime (operating temperature is critical for biological applications). (iii) Paraffin-based microvalves enable further reduction of the actuator volume unlike microvalves not making use of phase change (Pitchaimani et al. 2009).

Problems associated with incoherent solidification and incomplete retractions from the valve cavity are addressed by using a very shallow spherical and smooth valve seat which adopts the natural shape of a pressurized membrane. (The cavity was produced by laser ablation with a sophisticated scanning strategy).

Measuring the temperature of the heater during actuation would allow feedback control of the heater current for optimized performance. The valve can be used in real applications such as PCR amplification and in microfluidic networks including mixers that require time-dependent flow control. The overall thickness of the valve can be easily reduced by a factor of 3 for more bendability of the valve. This also implies smaller channel dimensions and therefore smaller stroke. A thinner valve can be fabricated in existing reel to reel processes (Vig et al. 2011). The time constant can be decreased by increasing the areal space for the actuation system. Work is in progress with an industrial partner to explore adhesive bonding techniques allowing higher pressures and automated assembly of the lab-on-foil microvalve suitable for industrial scale.
Acknowledgments The project is funded by the Dutch government through SenterNovem IOP project IPT06210B. The help from Henri Fledderus in the Nd:YAG laser cutting is acknowledged.

Open Access This article is distributed under the terms of the Creative Commons Attribution Noncommercial License which permits any noncommercial use, distribution, and reproduction in any medium, provided the original author(s) and source are credited.

\section{References}

Abgrall P, Gué A-M (2007) Lab-on-chip technologies: making a microfluidic network and coupling it into a complete microsystem-a review. J Micromech Microeng 17(5):R15-R49

Abgrall P, Lattes C, Conedera V, Dollat X, Colin S, Gue AM (2006) A novel fabrication method of flexible and monolithic 3D microfluidic structures using lamination of SU-8 films. J Micromech Microeng 16(1):113-121

Boden R, Lehto M, Simu U, Thornell G, Hjort K, Schweitz JA (2006) A polymeric paraffin actuated high-pressure micropump. Sens Actuator A Phys 127(1):88-93

Boustheen A, Homburg FGA, Bullema JE, Dietzel A (2008) Active microvalves for micro-fluidic networks in plastics-selecting suitable actuation schemes. In: 4th International Conference Multi-Material Micro Manufacture (4M2008), Cardiff, pp 317-320

Chow WWY, Lei KF, Shi G, Li WJ, Huang Q (2006) Microfluidic channel fabrication by PDMS-interface bonding. Smart Mater Struct 15(1):S112-S116

Flachsbart BR, Wong K, Iannacone JM, Abante EN, Vlach RL, Rauchfuss PA, Bohn PW, Sweedler JV, Shannon MA (2006) Design and fabrication of a multilayered polymer microfluidic chip with nanofluidic interconnects via adhesive contact printing. Lab Chip 6(5):667-674

Focke M, Kosse D, Muller C, Reinecke H, Zengerle R, Fv Stetten (2010) Lab-on-a-foil: microfluidics on thin and flexible films. Lab Chip 10(11):1365-1386

Han K-H, McConnell RD, Easley CJ, Bienvenue JM, Ferrance JP, Landers JP, Frazier AB (2007) An active microfluidic system packaging technology. Sens Actuator B Chem 122(1):337-346

Iverson B, Garimella S (2008) Recent advances in microscale pumping technologies: a review and evaluation. Microfluid Nanofluid 5(2):145-174

Jensen MF, McCormack JE, Helbo B, Christensen LH, Christensen TR, Geschke O (2004) Rapid prototyping of polymer microsystems via excimer laser ablation of polymeric moulds. Lab chip 4(4):391-395

Kaigala GV, Hoang VN, Backhouse CJ (2008) Electrically controlled microvalves to integrate microchip polymerase chain reaction and capillary electrophoresis. Lab Chip 8(7):1071-1078

Klintberg L, Svedberg M, Nikolajeff F, Thornell G (2003) Fabrication of a paraffin actuator using hot embossing of polycarbonate. Sens Actuator A Phys 103(3):307-316

Laser DJ, Santiago JG (2004) A review of micropumps. J Micromech Microeng 14(6):R35-R64

Lee JS, Lucyszyn S (2005) A micromachined refreshable braille cell. J Microelectromech Syst 14(4):673-682

Lee KS, Ram RJ (2009) Plastic-PDMS bonding for high pressure hydrolytically stable active microfluidics. Lab Chip 9(11):1618 1624

Lee JN, Jiang X, Ryan D, Whitesides GM (2004) Compatibility of mammalian cells on surfaces of poly(dimethylsiloxane). Langmuir 20(26):11684-11691 
Naessens K, Ottevaere H, Baets R, Daele PV, Thienpont H (2003) Direct writing of microlenses in polycarbonate with excimer laser ablation. Appl Optics 42(31):6349-6359

Oh KW, Ahn CH (2006) A review of microvalves. J Micromech Microeng 16(5):R13-R39

Pal R, Yang M, Johnson BN, Burke DT, Burns MA (2004) Phase change microvalve for integrated devices. Anal Chem 76(13): 3740-3748

Pham D, Tonge L, Cao J, Wright J, Papiernik M, Harvey E, Nicolau D (2002) Effects of polymer properties on laser ablation behaviour. Smart Mater Struct 11(5):668-674

Pitchaimani K, Sapp BC, Winter A, Gispanski A, Nishida T, Fan ZH (2009) Manufacturable plastic microfluidic valves using thermal actuation. Lab Chip 9(21):3082-3087

Selvaganapathy P, Carlen ET, Mastrangelo CH (2003) Electrothermally actuated inline microfluidic valve. Sens Actuator A Phys 104(3):275-282

Song WH, Kwan J, Kaigala GV, Hoang VN, Backhouse CJ (2008) Readily integrated, electrically controlled microvalves. J Micromec Microeng 18(4):045009

Suk JW, Jang JY, Cho JH (2008) Reagent-loaded plastic microfluidic chips for detecting homocysteine. J Micromech Microeng 18(5):055024. doi:10.1088/0960-1317/18/5/055024

Tang L, Lee NY (2010) A facile route for irreversible bonding of plastic-PDMS hybrid microdevices at room temperature. Lab Chip 10(10):1274
Tennico YH, Koesdjojo MT, Kondo S, Mandrell DT, Remcho VT (2010) Surface modification-assisted bonding of polymer-based microfluidic devices. Sens Actuators B: Chem 143(2):799-804

unkara V, Park DK, Hwang H, Chantiwas R, Soper SA, Cho YK et al (2011) Simple room temperature bonding of thermoplastics and poly(dimethylsiloxane). Lab Chip 11(5):962

van den Brand J, de Baets J, van Mol T, Dietzel A (2008) Systems-infoil-devices, fabrication processes and reliability issues. Microelectron Reliab 48(8-9):1123-1128

Vig AL, Mäkelä T, Majander P, Lambertini V, Ahopelto J, Kristensen A (2011) Roll-to-roll fabricated lab-on-a-chip devices. J Micromech Microeng 21(3):035006

Vlachopoulou ME, Tserepi A, Pavli P, Argitis P, Sanopoulou M, Misiakos K (2009) A low temperature surface modification assisted method for bonding plastic substrates. J Micromech Microeng 19(1):015007

Walsh D, Zoller P (1995) Standard Pressure Volume Temperature Data for Polymers. 1st ed. CRC Press

Woias P (2005) Micropumps-past, progress and future prospects. Sens Actuators B Chem 105(1):28-38

Yang B, Lin Q (2007) A latchable microvalve using phase change of paraffin wax. Sens Actuators A Phys 134(1):194-200

Yoo JC, Choi YJ, Kang CJ, Kim YS (2007) A novel polydimethylsiloxane microfluidic system including thermopneumatic-actuated micropump and Paraffin-actuated microvalve. Sens Actuators A Phys 139(1-2):216-220 